\title{
P16/Ki67 Dual Immunostaining in Conventional Cytology in Women with Positive Papanicolau Test
}

Agnieszka Korolczuk ${ }^{1 *}$, Monika Orzeł ${ }^{2}$, Sławomir Woźniak ${ }^{3}$, Agata Smoleń ${ }^{4}$ and Kinga Caban ${ }^{1}$

${ }^{1}$ Department of Clinical Pathomorphology, Medical University, Lublin, Poland

${ }^{2}$ Cormay SA, Lublin, Poland

${ }^{3}$ Department of Gynecology, Medical University, Lublin, Poland

${ }^{4}$ Department of Epidemiology, Medical University, Lublin

\begin{abstract}
Cytology-based screening has limited sensitivity to detect cervical precancerous lesion. Hihg risk human papilloma virus (HR HPV) DNA tests increase the detection of CIN II+ lesions, however it cannot differentiate if the infection is or transient or persistent. Its use is often limited by the need of standardisation of laboratory technics and relatively high costs.

Recently, several studies analysed the diagnostic performance of $\mathrm{p} 16 / \mathrm{Ki} 67$ dual-stained cytology as the possible alternative or coexisting option to HR HPV testing. In most of them the smear is obtained for liquid-based cytology.

This study was designed to analyze the usefulness of p16/Ki67 dual immunostaining in conventional cytology, which is the method of choice in cervical cancer screening in our country.

68 women with positive Pap test results were selected. Colposcopy, cervical biopsy and HR HPV DNA tests were performed in all patients. Separate slides for p16/Ki67 were collected at the time of colposcopy. Statistical analysis was performed to establish sensitivity, specificity, positive predictive value, negative predictive value and accurancy for all used tests. Biopsy-confirmed CIN was used as a gold standard for study purposes.

The diagnostic accurancy of p16/Ki67 dual-staining was the highest among used tests. Sensitivity of this test to detect CINII+ lesion was comparable to and specificity was higher than for HR HPV test. Similar results were observed for cytologic categories: LSIL and ASCUS. These results suggest that $\mathrm{p} 16 / \mathrm{Ki} 67$ immunostaining performed on conventional smears may become alternative or additional to HPV tests, due to its low costs and relatively simple procedure.
\end{abstract}

Keywords: Cervical intraepithelial neoplasia; CIN; Pap test; Dualstain cytology; p16 ${ }^{\mathrm{INK} 4 \mathrm{a}} ; \mathrm{Ki} 67$

\section{Introduction}

The screening programs based on cytology markedly reduced the incidence of cervical carcinoma. Pap test on its own still remains with relatively low sensitivity and high false-negative rates $[1,2]$. Introduction of liquid based cytology improved the accuracy of screening. Over the past years the causative role of Human Papillomavirus, high-risk types (HR-HPV), in the pathogenesis of cervical cancer, has been established [3]. Most HR-HPV infections are usually transient and may regress. In about $10 \%$ of these infections the process persists and progresses to high-grade squamous intraepithelial lesion [4]. Most of these persistent infections are localized within the transitional zone of the cervix [5]. The virus enters cells of the basal layer where alters the cell cycle by fusion of E6 and E7 viral oncogenes with nuclear cell-cycle regulatory proteins. This process results in overexpression of proteins such as p16 and Ki67, known biomarkers of neoplastic transformation of cells $[6,7]$. The protein p16 is increased as the consequence of inactivation of tumour suppressor function of $\mathrm{Rb}$ (Retinoblastoma) gene. Ki67 is known marker of cell proliferation, its expression within the squamous epithelium should be limited only to basal cells [8-10].

HPV DNA tests appear very useful in a management of women with positive cytology. However many clinical studies demonstrate that specificity of these tests is not significant for high-grade cervical lesion $[2,11,12]$, as it does not differentiate between transient and persistent HPV infection. HPV tests are not the best tool in women under 30 years of age as the frequency of HPV infection is very high [9,12-14]. In many countries the screening programs employ HPV tests over the age of 30 [15].
The problem remains where atypical squamous cells of undetermined significance (ASCUS) are diagnosed as the rate of this result is comparable in conventional and liquid based cytology. Approximately $10 \%$ of these women have underlying high-grade lesion [4]. All patients confronted with the diagnosis of ASCUS require careful clinical work-up to exclude high-grade intraepithelial lesion. In most, but not in all European countries, HPV tests are performed in patients with cytologic diagnosis of ASCUS. If the test is negative, the patient should be followed with co-testing at 3 years [15]. If the test is not performed, the serial cytology option incorporates cytology at 12 months, and if negative, cytology every 3 years [15]. Similar management is incorporated when LSIL (Low Grade Squamous Intraepithelial Lesion) is diagnosed. Polish National Health Service offers only repeated cytology at 6 months in all cases diagnosed with ASCUS and LSIL. This screening program is based on conventional cytology.

Recently, several studies analysed the diagnostic performance of

*Corresponding author: Agnieszka Korolczuk, Department of Clinical Pathomorphology, Medical University of Lublin, 20-059 Lublin, Jaczewskiego 8 , Poland, Tel: +48698982758; E-mail: agnieszka.korolczuk@wp.pl

Received August 03, 2015; Accepted August 27, 2015; Published August 29, 2015

Citation: Korolczuk A, Orzeł M, Woźniak S, Smoleń A, Caban K (2015) P16/ Ki67 Dual Immunostaining in Conventional Cytology in Women with Positive Papanicolau Test. J Cytol Histol 6: 358. doi:10.4172/2157-7099.1000358

Copyright: (C) 2015 Korolczuk A, et al. This is an open-access article distributed under the terms of the Creative Commons Attribution License, which permits unrestricted use, distribution, and reproduction in any medium, provided the original author and source are credited. 
p16/Ki67 dual-stained cytology as the possible alternative or coexisting option to HR HPV testing.

The aim of this study was to evaluate whether p16/Ki67 dualstained cytology, a biomarker combination indicative of transforming HPV infection, can provide high sensitivity and specificity for cervical precancerous lesion (CIN, Cervical Intraepithelial Neoplasia) in conventional cytology. Results were compared with HPV testing and colposcopy.

\section{Material and Methods}

98 women were included in this study. Group A consisted of 68 women, which in routine cytology-based cervical cancer screening were diagnosed with positive Pap test. The mean age of these patients was 41 (from 21 to 80 years of age). Group B consisted of 30 women (mean age 38: from 18 to 75 years of age) in which Pap test was negative.

All women did not have any previous history of gynaecological problems. In all patients colposcopy was performed. Separate conventional smear for p16/Ki67 dual stained cytology was obtained at the time of colposcopy. In women with positive Pap test (group A) cervical biopsy was collected for histopathological examination. These patients received HPV testing.

Pap cytology was interpreted in local cytology laboratories using the Bethesda System for reporting cervical cytology. Dual-stained cytology was performed in the Department of Clinical Pathomorphology (Medical University of Lublin) using CINtech PLUS kit (Roche mtm laboratories, Mannheim, Germany) according to the manufacture's instructions. One histopathologist, who was informed about patient's age but was blinded to all other study results, reviewed each slide.

One gynaecologist performed colposcopy according to accepted diagnostic standards. In line with current clinical practice, colposcopist was aware of Pap cytology and HPV test results. Cervical biopsy was taken at the time of colposcopy in women with positive Pap test results. Tissue samples were fixed in formalin and processed to paraffin blocks in the histopathological laboratory. $4 \mu \mathrm{m}$ slides were cut on the microtome, stained with hematoxylin and eosin $(\mathrm{H}+\mathrm{E})$ and assessed by one histopathologist. Biopsy-confirmed CIN was used as a gold standard for study purposes.

HPV test was performed in women with positive cytology results. The material for this test was collected at the time of colposcopy. Linear Array HPV Genotyping Test typing DNA of 37 viral subtypes (Roche Molecular Diagnostics, USA) was used.

\section{Statistical analysis}

We characterized the values of analysed parameters using frequencies and percentage due to the nominal scale of measurement.
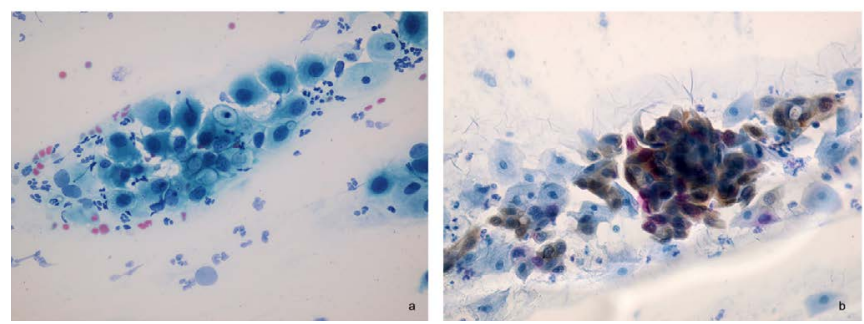

Figure 1: ASCUS cytology: a - Papanicolau staining. A typical Cells of Undetermined significance (ASCUS). b - Dual-stained cytology, red nuclear expresion of Ki67 and cytoplasmic brown expresion of p16 within dysplastic cells.

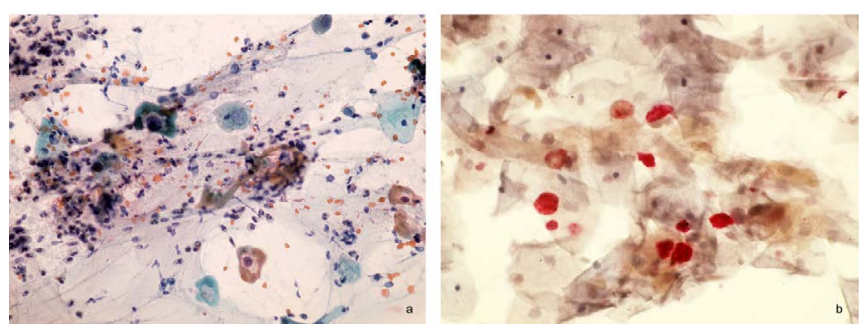

Figure 2: LSIL cytology: a - Papanicolau staining. Low Grade Squamous Intraepithelial Lesion, koilocytes with enlarged nuclei and perinuclear halo visible. b - p16/Ki67 immunostaining, dysplastic cells visible.
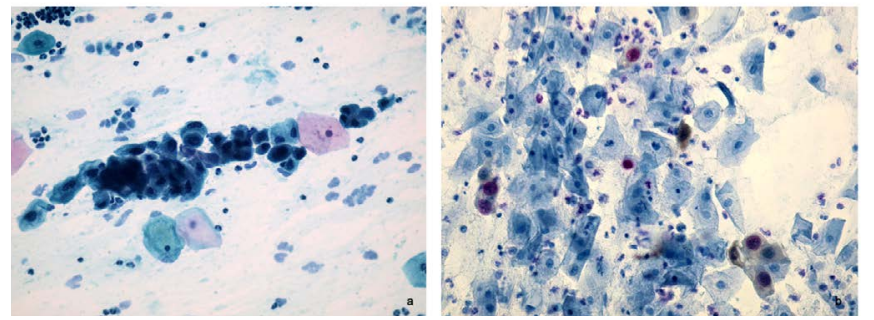

Figure 3: ASCH cytology: a-Papanicolau staining, Atypical cells, cannot exclude HSIL. b-p16/Ki67 immunostaining, positive dysplastic cells visible.
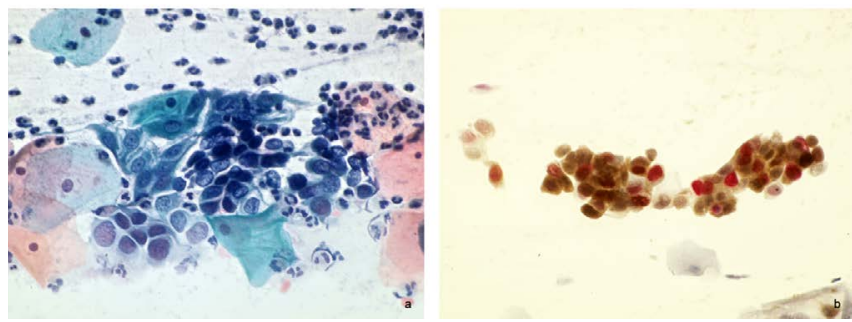

Figure 4: HSIL cytology: a- Papanicolau staining, High Grade Squamous Intraepithelial Lesion. b - p16/Ki67 immunostaining, dysplastic cells visible.

To evaluate the existence of differences or relationships between analysed categorical parameters cross-tabulation tables and the test for homogeneity or independence $\chi^{2}$ were used. In addition, the usefulness of the diagnostic value of: colposcopy, HPV testing and immunohistochemistry were evaluated.

The accuracy of the test was determined by a comparison between the results of the test and histopathology, which describes the actual disease state. Used basic measurements of accuracy of the test are as follows: sensitivity (SENS), specificity (SPEC), positive predictive value (PPV), negative predictive value (NPV), accurancy (ACC).

We accepted $5 \%$ error of inference and the associated significance level of $\mathrm{p}$ value $<0.05$ indicating the existence of statistically significant differences or relationships. The statistical analyses were performed using computer software STATISTICA v. 10.0 (StatSoft, USA).

\section{Results}

In all women of group B Pap test did not show any abnormalities of squamous cervical epithelium. In Group A ASCUS was diagnosed in 7 women (Figure 1a), in 2 women ASC-H- atypical squamous cellscannot exclude HSIL (Figure 2a), in 28 - LSIL (CIN I/HPV) (Figure 3a), and in 31 - HSIL (CIN II/CIN III) (Figure 4a). 
Citation: Korolczuk A, Orzeł M, Woźniak S, Smoleń A, Caban K (2015) P16/Ki67 Dual Immunostaining in Conventional Cytology in Women with Positive Papanicolau Test. J Cytol Histol 6: 358. doi:10.4172/2157-7099.1000358

Colposcopy was satisfactory in 61 women of group A. In 54 women of this group colposcopy showed abnormalities that were confirmed by histopathology. In 7 women with biopsy confirmed CIN, colposcopy gave negative results.

Microscopic examination of cervical biopsy confirmed the presence of CIN in 5/7 ASCUS, 2/2 ASC-H, 21/28LSIL and 31/31 HSIL patients.

HR HPV was present in 3/7women diagnosed with ASCUS, 2/2 diagnosed with ASC-H, 21/28 diagnosed with LSIL and 25/31 diagnosed with HSIL.

\section{P16/Ki67 immunocytochemistry}

P16/Ki67 expression was negative in all women of group B. The week cytoplasmic expression of p16 was observed in a few cases in glandular cervical and metaplastic cells.

Positive results were present in women of group A. Positive cells showed red nuclear expression of Ki67 and brown nuclear and/or cytoplasmic expression of p16. The positive cells were present in $3 / 7$ women diagnosed with ASCUS (Figure 1b), 2/2 diagnosed with ASC-H (Figure 2b), 18/28 diagnosed with LSIL (Figure $3 \mathrm{~b}$ ) and in $31 / 31$ diagnosed with HSIL (Figure 4b).

10 smears collected from women diagnosed with LSIL showed no p16/Ki67 positive cells. Two of these patients showed the presence of HR HPV and CIN positive biopsy. One of these smears collected for dual staining was of low cellularity and showed no presence of glandular cells, the second one presented advanced cytolysis.

In women of group A, within smears that were collected separately for p16/Ki67, we observed the presence of slides with numerous neutrophils or erythrocytes. However in all these smears cells with p16/ Ki67 expression were present.

\section{Diagnostic accuracy of all examined tests}

Results of statistical analysis show that sensitivity of HR HPV test and dual staining p16/Ki67 was high (0,86 each respectively) and specificity was the highest of all examined tests $(0.89$ and 1.0 respectively). Sensitivity of colposcopy was the highest $(0.88)$ but specificity of this test was low (0.11) (Table 1$)$.

\begin{tabular}{|l|c|c|c|c|c|c|c|c|c|}
\hline & TP & FP & FN & TN & SENS & SPEC & PPV & NPV & ACC \\
\hline HPV HR & 51 & 1 & 8 & 8 & 0,86 & 0,89 & 0,98 & 0,50 & 0,87 \\
\hline P16/Ki67 & 51 & 0 & 8 & 9 & 0,86 & 1,00 & 1,00 & 0,53 & 0,88 \\
\hline colposcopy & 52 & 8 & 7 & 1 & 0,88 & 0,11 & 0,87 & 0,13 & 0,78 \\
\hline
\end{tabular}

TP - true positive results; FP - false positive results; FN - false negative results TP - true negative results; SENS - sensitivity, SPEC - specificity, PPV - positive predictive value, NPV - negative predictive value, ACC - accurancy.

Table 1: Results of statistical analysis.

\section{ASCUS+ASCH}

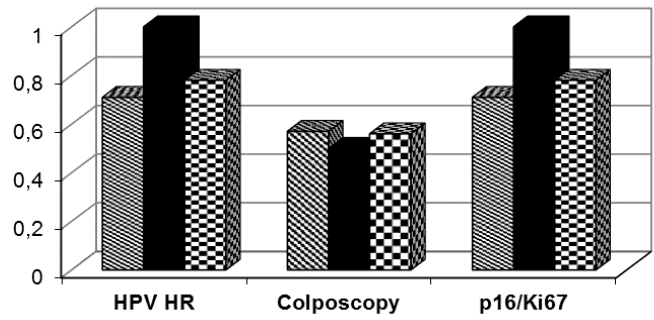

Figure 5: Diagnostic accuracy of examined tests in group of cytological diagnosis: ASCUS+ASC-H.

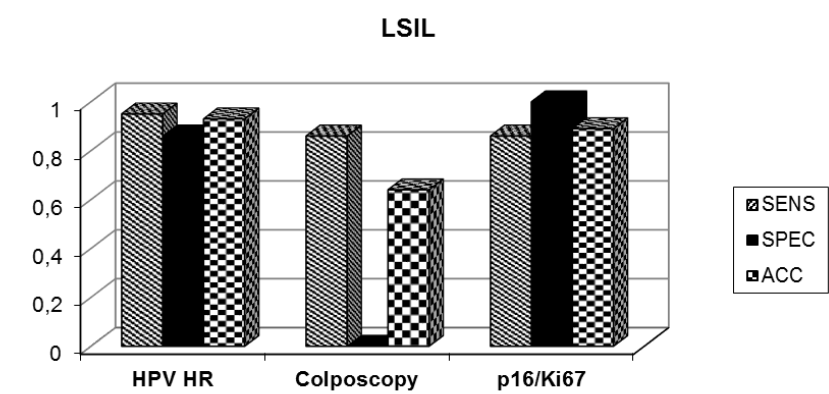

Figure 6: Diagnostic accuracy of examined tests in group of cytological diagnosis: LSIL.

\begin{tabular}{|l|c|c|c|c|c|c|c|c|c|}
\hline CIN II/CINIII & TP & FP & FN & TN & SENS & SPEC & PPV & NPV & ACC \\
\hline HPV HR & $15 / 17$ & $1 / 1$ & $1 / 2$ & $8 / 8$ & $0,94 / 0,89$ & $0,89 / 0,89$ & $0,94 / 0,94$ & $0,89 / 0,8$ & $0,92 / 0,89$ \\
\hline P16/Ki67 & $15 / 19$ & $0 / 0$ & $1 / 0$ & $9 / 9$ & $0,94 / 1,00$ & $1,00 / 1,00$ & $1,00 / 1,00$ & $0,90 / 1,00$ & $0,96 / 1,00$ \\
\hline colposcopy & $16 / 18$ & $8 / 8$ & $0 / 1$ & $1 / 1$ & $1,00 / 0,95$ & $0,11 / 0,11$ & $0,67 / 0,69$ & $1,00 / 0,5$ & $0,68 / 0,68$ \\
\hline
\end{tabular}

TP - true positive results; FP - false positive results; FN - false negative results; TP - true negative results; SENS - sensitivity, SPEC - specificity, PPV - positive predictive value, NPV - negative predictive value, ACC - accurancy

Table 2: Diagnostic accuracy of examined tests for CIN II+

HR HPV and p16/Ki67 were characterized by the highest negative and positive predictive values (NPV, PPV) (Table 1).

We also analysed the above parameters separately for groups of cytological diagnosis of: LSIL, and ASCUS+ASC-H. Due to a small number of cases of ASCUS and ASC-H, we treated them as a one group in this part of our analysis.

The last step of our analysis was to establish the diagnostic accuracy of the used tests for CIN II and CIN III.

For LSIL and ASCUS+ASCH the values of sensitivity and specificity were the highest for HR HPV and p16/Ki67 (Figures 5 and 6). The best diagnostic values of examined parameters to detect CIN II and CIN III were for dual-staining p16/Ki67 and HR HPV test (Table 2).

\section{Discussion}

This study was performed to assess the results of p16/Ki67 dual staining in women with positive Pap tests. We also analysed the diagnostic values of p16/Ki67, HR HPV test and colposcopy. As the true disease state results of microscopic examination of cervical biopsy were established. Cytology as well as p16/Ki67 were performed on conventional slides, as this method is the most common in our country. This is associated with low costs of the test, but also with the fact, that liquid-based cytology is available only in a few laboratories located in big cities.

HR HPV tests significantly increased the sensitivity for the detection of CINII/CINIII when used as an adjunctive test to cytology or as primary screening method. The use of these tests lets to extend the breaks among the Pap tests [16]. However positive HR HPV test cannot answer if the infection is transient or persistent [17]. The usefulness of HR HPV test in Low Grade lesions (ASCUS and LSIL) is low [18]. Most of HPV infections are transient and have tendency to regress $[6,19,20]$. In women with positive HR HPV further procedures have to be employed: repeated cytology, colposcopy or/and cervical biopsy [7]. The use of mRNA HR HPH tests increases the detection of CIN II+ lesions, but its use is often limited by the need of standardisation of laboratory technics and high costs of the test. 
The overexpression of p16 in cervical dysplasia is associated with the transforming activity of the E7 oncoprotein of HR HPV types and can be regarded as a marker of the E7-mediated inactivation of the tumorsupressor function of the retinoblastoma protein $(\mathrm{pRb})$. Therefore, simultaneous detection of p16 overexpression and expression of the proliferation marker Ki67 within the same cervical epithelial cells gives the possibility of identifying cells with true oncogenic transformation [7]. As the HSIL is known of high sensitivity in CIN II+ detection, the problem remains in LSIL and ASCUS patients. Only a few published studies have described the results of p16/Ki67 dual immunostaining in cervical cytology in these groups of patients. Bergeron et al. [21] performed the results of cytology-based screening in a group of 28 thousand women from 5 European countries. In women with the diagnosis of ASCUS and LSIL the p16/Ki67 dual-staining as well as HR HPV test were performed. p16/Ki-67 sensitivity in ASCUS for the detection of CIN II+ was 92.2\% (HR HPV test - 90.9\%), specificity was $80.6 \%$ (HR HPV test $-36.3 \%$ ). In a group of cytological diagnosis of LSIL sensitivity was $94.2 \%$ (96.4\% for HR HPV), specificity was 68.0\% (19.1\% for HR HPV). Schmidt et al. [22] evaluated the diagnostic performance of dual immunostaining in CIN II+ detection with ASCUS and LSIL Pap smears. These authors reported a sensitivity of $92.2 \%$ (ASCUS) and 94.2 (LSIL) and a specificity of $80.6 \%$ (ASCUS) and $68.0 \%$ (LSIL). Petry et al. [10] evaluated the utility of this test for the identification of CIN II+ lesions in women with Pap negative and HR HPV positive screening results. Sensitivity of p16/Ki67 for the detection of biopsy-confirmed CIN II+ was $91.9 \%$ for CIN II and $96.4 \%$ for CIN III, while specificity was $82.1 \%$ for CIN II on biopsy and 76.9 for CIN III respectively. Wentzensen et al. [23] compared the results of HR HPV test with p16/Ki67 dual immunostaining in detection of CIN II+ lesions in a high risk colposcopy clinic. Sensitivity and specificity of dual stained cytology was $85.5 \%$ and $59.4 \%$ respectively for CIN II and $90.6 \%$ and $48.6 \%$ respectively for CIN III.

The results of the above mentioned studies indicate that p16/Ki67 dual-stained cytology may identify women with a high probability of underlying CIN II+ and may substitute for HR HPV triage. Dual immunostaining was performed on liquid based cytology in all these studies.

To investigate whether the use of cervical cancer biomarkers can improve the detection of CIN II+ in conventional cytology, we performed p16/Ki67 dual immunocytochemical stain on conventional smears and compared the results with HR HPV test and colposcopy in women with positive Pap test results.

Conventional cytology is known to have many limits. The first and the most important one is its adequacy and quality. The microscopic assessment may be limited by advanced cytology, low cellularity, lack of glandular cervical cells or the presence of numerous erythrocytes and/ or inflammatory cells. In cytology-based screening programs it still remains the method of choice in many countries, what is associated with its low costs. In Poland, National Health Service screening program based on conventional cytology offers for women with the cytological diagnosis of ASCUS repeated Pap test (within 6 months) and for women with LSIL- colposcopy and repeated cytology (6 months). The potential performance of HR HPV tests is limited mainly by its high costs but also by the fact that not many laboratories have standardised methods to run these tests.

Our results show that sensitivity of p16/Ki67 dual-stained cytology for ASCUS+ASC-H was 0.71 and for LSIL was 0.86 and was equal or comparable with sensitivity of HR HPV test (0.71 for ASCUS+ASC-H and 0.96 for LSIL). Specificity of p16/Ki67 was high: 1.0 for
ASCUS+ASC-H group and 1.0 for LSIL; in this group the examined parameter was higher than HR HPH test (0.86). Dual-stained cytology had the highest accuracy in the detection of CIN II+ lesions: 0.96 for CIN II and 1.0 for CIN III. Positive predictive value (PPV) and negative predictive value (NPV) for the detection of CIN II+ were the highest for p16/Ki67 immunostaining: PPV was 1.0 for CIN II and CIN III and NPV was 0.9 and 1.0 for CIN II and CIN III respectively, when comparing to colposcopy (PPV 0.69 and NPV 0.50) and HR HPV (PPV 0.94 and NPV 0.80). This data indicate the high diagnostic accurancy of our immunostaining in this study.

In most of the studies immunocytochemical dual-staining for $\mathrm{p} 16 /$ Ki67 is performed on liquid based cytology [1,7,21,22,24-27]. In our study we assessed expresion of p16/Ki67 in conventional cytology, which is the basic method recomended in our country by Nathional Health Service screening program for cervical cancer. The quality of slides in liquid-based cytology is much better than in conventional smears. Still, in studies that use liquid-based cytology, authors report cases, in which the false negative result is due to quality of smears, mainly to their low cellularity $[1,8]$. We have also noticed the presence of false negative results in women with positive cytology and histology. In all these slides we have not seen cells with positive reaction for $\mathrm{p} 16 /$ Ki67 dual-staining. All of these smears were of poor quality, mainly due to low cellularity or advanced cytolysis. It is worth to mention here, that in slides with numerous inflammatory cells or erythrocytes, we have observed the presence of p16/Ki67 positive squamous epithelial cells (Figure 3b).

In conclusion, we may say that immunocytochemical dual staining for $\mathrm{p} 16 / \mathrm{Ki} 67$ performed on conventional smears may become additional to HPV tests. If there is not possibility to perform HPV test, dual staining could help to determine further management with the patient. Due to its low costs ( 2 to 3 times lower than HPV test) and relatively simple procedure that can be used in most of cytological laboratories, it may become an essential method for women with positive Pap test results, especially ASCUS or LSIL, in everyday practice. The employment of dual-stained immunocytochemistry especially in women with ASCUS could be considered in the national guidelines. Offering to the patient diagnosed with ASCUS the possibility of more precise diagnosis: positive or negative, might lead to shorten her stress associated with positive Pap test result and also shorten the waiting time for the treatment. Our results need further verification by studies involving large number of patients.

\section{References}

1. Sahebali S, Depuydt CE, Boulet GA, Arbyn M, Moeneclaey LM, et al. (2006) Immunocytochemistry in liquid-based cervical cytology: analysis of clinical use following a cross-sectional study. Int J Cancer 118: 1254-1260.

2. Ikenberg H, Bergeron C, Schmidt D, Griesser H, Alameda F, et al. (2013) Screening for cervical cancer precursors with $\mathrm{p} 16 / \mathrm{Ki}-67$ dual-stained cytology: results of the PALMS study. J Natl Cancer Inst 105: 1550-1557.

3. Hausen HZ (2002) Papillomaviruses and cancer: from basic studies to clinical application. Nat Rev Cancer 2: 342-350.

4. von Knebel Doeberitz M, Reuschenbach M, Schmidt D, Bergeron C (2012) Biomarkers for cervical cancer screening: the role of p16(INK4a) to highlight transforming HPV infections. Expert Rev Proteomics 9: 149-163.

5. Schiffman M, Castle PE, Jeronimo J, Rodriguez AC, Wacholder S (2007) Human papillomavirus and cervical cancer. Lancet 370: 890-907.

6. Stanley M (2010) Pathology and epidemiology of HPV infection in females Gynecol Oncol 117: S5-10.

7. Killeen JL, Dye T, Grace C, Hiraoka M (2014) Improved abnormal Pap smear triage using cervical cancer biomarkers. J Low Genit Tract Dis 18: 1-7. 
Citation: Korolczuk A, Orzeł M, Woźniak S, Smoleń A, Caban K (2015) P16/Ki67 Dual Immunostaining in Conventional Cytology in Women with Positive Papanicolau Test. J Cytol Histol 6: 358. doi:10.4172/2157-7099.1000358

8. Trunk MJ, Dallenbach-Hellweg G, Ridder R, Petry KU, Ikenberg H, et al. (2004) Morphologic characteristics of p16INK4a-positive cells in cervical cytology samples. Acta Cytol 48: 771-782.

9. Hwang SJ, Shroyer KR (2012) Biomarkers of cervical dysplasia and carcinoma J Oncol 2012: 507286

10. Petry KU, Schmidt D, Scherbring S, Luyten A, Reinecke-Lüthge A, et al. (2011) Triaging Pap cytology negative, HPV positive cervical cancer screening results with p16/Ki-67 Dual-stained cytology. Gynecol Oncol 121: 505-509.

11. Lörincz AT, Richart RM (2003) Human papillomavirus DNA testing as an adjunct to cytology in cervical screening programs. Arch Pathol Lab Med 127: 959-968.

12. Denton KJ, Bergeron C, Klement P, Trunk MJ, Keller T, et al. (2010) The sensitivity and specificity of $\mathrm{p} 16$ (INK4a) cytology vs HPV testing for detecting high-grade cervical disease in the triage of ASC-US and LSIL pap cytology results. Am J Clin Pathol 134: 12-21.

13. Wright JD, Rader JS, Davila R, Powell MA, Mutch DG, et al. (2006) Human papillomavirus triage for young women with atypical squamous cells of undetermined significance. Obstet Gynecol 107: 822-829.

14. Saslow D, Salomon D, Lawson HW, Killackey M, Kulasingam SL, et al. (2012) American Cancer Society, American Society for Colposcopy and Cervical Pathology, and American Society for Clinical Pathology screening guidelines for the prevention and early detection of cervical cancer. CA Cancer J Clin 62 : 142-172.

15. Massad LS, Einstein MH, Huh WK, Katki HA, Kinney WK, et al. (2013) 2012 Updated Consensus Guidelines for the Management of Abnormal Cervical Cancer Screening Tests and Cancer Precursors. J Low Genit Tract Dis 17 $1-27$.

16. Dillner J, Rebolj M, Birembaut P, Petry KU, Szarewski A, et al. (2008) Long term predictive values of cytology and human papillomavirus testing in cervica cancer screening: joint European cohort study. BMJ 337: a1754.

17. Schiffman M, Wentzensen N (2010) From human papillomavirus to cervical cancer. Obstet Gynecol 116: 177-185.
18. Tsoumpou I, Arbyn M, Kyrgiou M, Wentzensen N, Koliopoulos G, et al. (2009) $\mathrm{p} 16$ (INK4a) immunostaining in cytological and histological specimens from the uterine cervix: a systematic review and meta-analysis. Cancer Treat Rev 35 210-220.

19. Sahasrabuddhe VV, Luhn P, Wentzensen N (2011) Human papillomavirus and cervical cancer: biomarkers for improved prevention efforts. Future Microbiol 6: 1083-1098

20. Woodman CB, Collins SI, Young LS (2007) The natural history of cervical HPV infection: unresolved issues. Nat Rev Cancer 7: 11-22.

21. Bergeron C, Schmidt D, Ikenberg H, Ridder R (2010) High sensitivity and high specificity of p16/Ki-67 dual-stained cytology for high-grade $\mathrm{CIN}$ - results from screening and triage trials in over 28000 women. Cancer Cytopathol 118: 305-306.

22. Schmidt D, Bergeron C, Denton KJ, Ridder R, European CINtec Cytology Study Group (2011) p16/ki-67 dual-stain cytology in the triage of ASCUS and LSIL papanicolaou cytology: results from the European equivocal or mildly abnormal Papanicolaou cytology study. Cancer Cytopathol 119: 158-166.

23. Wentzensen N, Schwartz L, Zuna RE, Smith K, Mathews C, et al. (2012) Performance of $\mathrm{p} 16 / \mathrm{Ki}-67$ immunostaining to detect cervical cancer precursors in a colposcopy referral population. Clin Cancer Res 18: 4154-4162.

24. Guo M, Hu L, Baliga M, He Z, Hughson MD (2004) The predictive value of p16(INK4a) and hybrid capture 2 human papillomavirus testing for high-grade cervical intraepithelial neoplasia. Am J Clin Pathol 122: 894-901.

25. Baak JP, Kruse AJ, Robboy SJ, Janssen EA, van Diermen B, et al. (2006) Dynamic behavioural interpretation of cervical intraepithelial neoplasia with molecular biomarkers. J Clin Pathol 59: 1017-1028.

26. Meyer JL, Hanlon DW, Andersen BT, Rasmussen OF, Bisgaard K (2007) Evaluation of p16INK4a expression in ThinPrep cervical specimens with the CINtec p16INK4a assay: correlation with biopsy follow-up results. Cancer 111 83-92.

27. Cuschieri K, Wentzensen N (2008) Human papillomavirus mRNA and p16 detection as biomarkers for the improved diagnosis of cervical neoplasia. Cancer Epidemiol Biomarkers Prev 17: 2536-2545. 